\title{
Evaluation of the out-of-plane behavior of stud-to-track connections in nonstructural partition walls
}

\author{
Esmaeel Rahmanishamsi ${ }^{1}$ a , Siavash Soroushian " , Emmanuel "Manos" \\ Maragakis $^{c}$ \\ ${ }^{a}$ Postdoctoral Fellow, Department of Civil and Environmental Engineering, University of Nevada, \\ Reno, Reno, NV, 89557, email: erahmanishamsi@unr.edu \\ b Structural Analyst, Advanced Technology and Research, Arup, San Francisco, CA, 94105, \\ email: siavash.soroushian@arup.com \\ ${ }^{\mathrm{c}}$ Professor, Dean of College of Engineering, University of Nevada, Reno, Reno, NV, 89557, email: \\ maragaki@unr.edu
}

\begin{abstract}
A series of component-level experiments have been performed aiming to characterize the out-of-plane force-displacement response and damage mechanisms of stud-to-track connections in nonstructural steel-framed partition walls. The performance of connections with various stud-to-track gap dimensions, stud and track thicknesses, and screw-attachment configurations were evaluated and compared. In addition, the accuracy of available design provisions for estimating the ultimate connection capacity was assessed. The experimental data was then used to generate capacity fragility curves in terms of displacement and force. Finally, a series of nonlinear numerical hinge models were developed and calibrated that represent the out-of-plane hysteresis behavior of stud-to-track connections.
\end{abstract}

Keywords: Nonstructural systems, Partition walls, Cold-formed steel, Stud-to-track connections, Fragility analysis, Numerical modeling

\section{Introduction}

Nonstructural systems almost always represent the major portion (approximately $48 \%$ to $70 \%$ ) of the total construction cost in buildings [1]. During an earthquake event, these

\footnotetext{
${ }^{1}$ Corresponding author
} 
systems are subjected to the dynamic environment of the building. However, they are rarely considered in current earthquake design methodology of new buildings [2]. Consequently, it is not surprising that recent earthquakes have demonstrated poor performance of nonstructural systems, resulting in significant economic loss, typically exceeding the economic loss associated with structural damage [3, 4, 5, 6, and 7]. Indeed, nonstructural systems account for over $78 \%$ of the total estimated national annualized earthquake loss [1].

Among various nonstructural systems, steel-framed gypsum partition walls represent a substantial contribution to the total investment in a building. These walls configure the architectural layout of a building, thereby facilitating its functionality for occupants [8]. Pervasive damage to partition walls has been observed in previous earthquakes. The damage was often initiated at shake intensities much lower than those causing structural damage [9]. Partition damage can lead not only to property loss, but also to loss of functionality of critical facilities, such as operating rooms in hospitals, which might be followed by fatalities, even in low or mid-intensity earthquakes.

A number of experimental studies were carried out in recent years in order to assess the in-plane and out-of-plane seismic performance of steel-framed partition walls $[10,11,12,13$, $2,14,15,16,17$, and 9]. These studies investigated the damage mechanisms and hysteresis behaviors of partition walls with different configurations. Where the return walls were included in the test, one of the observed damage mechanisms was damage to the stud-to-track connections in the out-of-plane direction. Retamales et al. [15] and Rahmanishamsi et al. [16] reported extensive deformation of track flanges of return walls. The deformation allowed return-wall studs to pop out from tracks. Moreover, the stud webs were crippled at the locations of some of the stud-to-track connections, when walls were subjected to extreme 
out-of-plane excitations. The research also showed that the out-of-plane stiffness and strength of partition walls depend on the characteristics of stud-to-track connections. Therefore, the behavior of the stud-to-track connections in the out-of-plane direction is of interest to determine its role in the performance of steel-framed gypsum partition walls.

Limited research has been conducted on the performance of stud-to-track connections in the out-of-plane direction. Compiling and analyzing the experimental data from a variety of sources, Fox and Schuster [18] recognized crippling of stud webs and punching-through of track flanges as dominant failure modes of stud-to-track connections. They also proposed design expressions to predict the connection capacities based on these failure modes. Bolte and LaBoube [19] expanded the available data with 24 additional tests. The specimens were different in terms of stud-to-track gaps, stud and track thicknesses, and whether studs were screwed to tracks (screw-attached configuration) or not (deflection-track configuration). The researchers compared the experimental results with the available design provisions, including AISI specification [20] and US Army Corps of Engineers technical instruction [21], and recommended some modifications. Moreover, the failure of the connection due to trackflange deformation was discussed. Recently, a comprehensive study was also conducted on the performance of jamb stud-to-track connections [22]. The previous studies provided valuable information on stud-to-track connections; nonetheless, they were limited to connections in load-bearing walls. The steel track and stud profiles in load-bearing walls are different from those used in nonstructural partition walls. Thinner track/stud profiles with smaller web depth are usually employed in nonstructural partition walls since they are not part of the structural load-carrying system [23]. In addition, according to best of the authors' 
knowledge, no study has evaluated the hysteresis force-displacement behavior of stud-totrack connections.

This study is aimed at addressing the missing information about the out-of-plane damage mechanisms and force-displacement characteristics of stud-to-track connections in nonstructural partition walls. For this purpose, a series of monotonic and cyclic experiments have been performed at the University of Nevada, Reno, as part of a grand challenge project titled "NEESR-GC: Simulation of the Seismic Performance of Nonstructural Systems." The test setup and experimental program are described in this paper, followed by an outline of the observed damage mechanisms. The force-displacement responses of connections with various stud and track thicknesses, stud-to-track gaps, and connection configurations (either screw-attached or deflection-track) are then compared. In addition, the correlation between the tested ultimate connection capacities with currently available design provisions was evaluated. Afterwards, the experimental data was utilized to generate capacity fragility curves in terms of displacement and force. Finally, a series of nonlinear numerical hinge models were proposed and calibrated using component experimental data to represent the hysteresis behavior of stud-to-track connections in the out-of-plane direction. Note that the long-term objectives of these models are to be used in conjunction with the numerical models of other wall components (such as gypsum-to-stud connections and steel studs) in order to develop a detailed numerical model of steel-framed gypsum partition walls [23].

\section{Description of test specimens}

\subsection{Test setup}

A sample specimen and the testing machine is presented in Fig. 1. The specimens

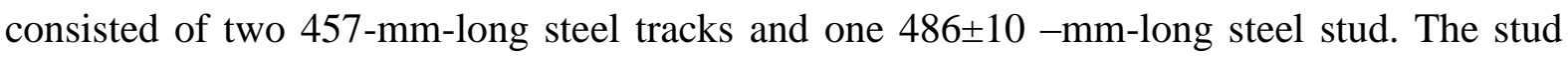


and tracks were either $0.48 \mathrm{~mm}$ thick (362S/T125-19) or $0.75 \mathrm{~mm}$ thick (362T125-30). These products were selected from the common construction details of nonstructural partition walls in commercial and institutional buildings [15]. The stud was nested into the tracks, which were bolted to vertical supports spaced $511 \mathrm{~mm}$ apart. The gap between the end of the stud and the web of the tracks (gap in Fig. 1b) was changed from $3 \mathrm{~mm}$ to $22 \mathrm{~mm}$. For some specimens two \#8 $\times 13$-mm screws were used to attach the stud to the tracks, while for others the screws were omitted to represent a deflection track configuration [21]. The vertical supports were fixed to the stationary base of an Instron 5985 machine. In order to prevent the bending of the stud and limit the deformation to stud-to-track connections, the middle of the stud was clamped between two $140 \times 89 \times 6$-mm steel plates. One of these plates was held by the movable grip of the machine. The machine applied upward and downward displacement to the specimens through the movable grip and measured the reaction force by the axial load cell (Fig. 1a).

\subsection{Experimental program}

A total of 26 specimens, categorized in six series, were tested to evaluate the strength and stiffness of stud-to-track connections in the out-of-plane direction (Table 1). The series were different in terms of the stud and track thicknesses, the stud-to-track gaps, and the stud-totrack connection configurations (screw-attached or deflection-track). An abbreviated nomenclature was adopted to label the series that described all these characteristics. Namely, the first group of characters denotes the stud and track thickness (T48: $0.48 \mathrm{~mm}$ thick), the second group of characters indicates the gap (G13: $13 \mathrm{~mm}$ gap), and the third group of characters stands for the connection configuration (SA: screw-attached and DT: deflectiontrack). For fragility assessment purposes, at least three nominally identical specimens were 
tested under cyclic loading for each series [15]. For cyclic tests, a loading protocol proposed by Retamales et al. [24] was adopted. The loading protocol was established for evaluating the performance of drift-sensitive nonstructural components. The input variables for the protocol include $\Delta_{\max }=50 \mathrm{~mm}$ : the maximum displacement imposed by the testing protocol, $\sigma=260$ second: a parameter that controls the shape of the testing protocol envelope, $f_{\max }-f_{\min }=0.17$ $0.011 \mathrm{~Hz}$ : the range of the frequencies, and $S_{r}=0.47$ octaves $/ \mathrm{min}$ : the sweep rate. Fig. 2 shows the displacement history that was generated based on this loading protocol and applied to the specimens. Additional monotonic tests were also performed in order to determine the effect of cumulative cyclic damage on the capacity of stud-to-track connections. The loading rate varied from $0.04 \mathrm{~mm} / \mathrm{sec}$ to $0.42 \mathrm{~mm} / \mathrm{sec}$ for the first series. However, a constant rate $(0.21 \mathrm{~mm} / \mathrm{sec})$ was used for the remaining tests since the response of the connection was found to be insensitive to the loading rate.

\section{Experimental results}

\subsection{Individual stud-to-track connection force and displacement}

The free-body diagram of force and displacement of the test setup in a downward monotonic test is provided in Fig. 3. It is assumed that the displacement of two stud-to-track connections are identical and equal to the displacement of the movable grip. In fact, the flexural deformation of the stud is considered to be negligible in comparison to deformation of stud-to-track connections. Moreover, the total force (P in Fig. 3) is assumed to be equally distributed between two connections. Thus, the force for an individual connection was calculated as P/2. 


\subsection{Damage mechanisms}

Fig. 4 depicts the basic behavior and damage mechanisms of a stud-to-track connection subjected to an increasing downward displacement. A slight track-flange deformation ( $S D$, deformation smaller than $10 \mathrm{~mm}$ ) was initially observed as the stud moved downward. For deflection-track configurations with large stud-to-track gaps (equal to or larger than $13 \mathrm{~mm}$ ), this damage was followed by an excessive track-flange deformation ( $E D$, deformation larger than $10 \mathrm{~mm}$ ) that led to the permanent displacement of the stud (PD) (Fig. 4c). For other specimens, increasing the downward displacement caused the crippling of the stud web (WC) (Fig. 4d). Where screws were used to attach the stud to tracks, the screws were subsequently pulled from the studs (SP) (Fig. 4e). All tests were continued until the studs were pushed out of the tracks, which was considered a complete failure of the connection. The last column of Table 2 (in section 3.3) summarizes the damage mechanisms observed in every test.

\subsection{Ultimate connection capacity}

The typical experimental force-displacement responses in monotonic and cyclic tests and displacement zones corresponding to each damage description are provided in Fig. 5. The maximum recorded force, called the ultimate tested capacity, was dominated by track deflection (ED), web crippling (WC), or a combination of web crippling and screw pull-out (SP). According to AISI S100 [25] the web crippling strength of studs can be computed as below:

$$
\text { Web crippling: } P_{n}=C t^{2} F_{y}\left(1-C_{R} \sqrt{R / t}\right)\left(1-C_{N} \sqrt{N / t}\right)\left(1-C_{h} \sqrt{h / t}\right)
$$

where $C$ : web crippling coefficient,$C_{R}$ : inside bend radius coefficient, $C_{N}$ : bearing length coefficient, $C_{h}$ : web slenderness coefficient, $t$ : stud thickness, $F_{y}$ : yield strength of the stud 
material, $R$ : stud inside bend radius, $N$ : stud bearing length, and $h$ : flat dimension of stud web. Considering “One-Flange Loading” condition and $R / t \leq 9$, the coefficients' values can be chosen from the table C3.4.1-1 of AISI S100 as follow, $C: 4, C_{R}: 0.14, C_{N}: 0.35$, and $C_{h}: 0.02$.

The AISI provision [25] also includes a design expression for nominal pull-out strength of a screw subjected to tension force [19], $P_{n o t}$,

$$
P_{\text {not }}=0.85 t_{c} d F_{u 2}
$$

where $d$ : nominal screw diameter, $F_{u 2}$ : tensile strength of the member not in contact with screw head, and $t_{c}$ : lesser of depth of penetration and thickness of the member not in contact with the screw head.

Using these provisions, Bolte and LaBoube 19] proposed the following equations to predict the capacity of stud-to-track connections with either deflection-track or screwattached configurations:

$$
\begin{aligned}
& \text { Screw-attached: } P_{n s t}=P_{n}+R \times P_{\text {not }} \\
& \text { Deflection-track: } P_{n d t}=b_{e f f} t^{2} F_{y} / 4 e
\end{aligned}
$$

where $R$ : a reduction factor, $e$ : the gap dimension, $t$ : the track thickness, $F_{y}$ : track material yield strength, and $b_{\text {eff: }}$ effective flange length. The effective flange length was defined as a function of stud-flange width $\left(w_{\text {stud }}\right)$ and the ratio of gap to track thickness:

$$
b_{e f f}=\Delta+w_{s t u d}
$$




$$
\Delta= \begin{cases}\frac{300\left(e / t^{2}\right)}{100} & \text { for }\left(e / t^{2}\right)<100 \\ 300 & \text { for }\left(e / t^{2}\right) \geq 100\end{cases}
$$

In this study, Eq. (3) with an $\mathrm{R}=1$ was employed to estimate the maximum capacity of specimens with screw-attached configuration. For other specimens, the lesser of the values calculated from Eqs. (1) and (4) were considered as the maximum capacity. In fact, both damage mechanisms $W C$ and $E D$ were checked to determine the mechanism that governed the capacity. Based on the manufacturer catalog, the following tensile strengths were used: for 0.75 -mm-thick studs/tracks, a tensile strength of $228 \mathrm{MPa}$; for 0.48 -mm-thick studs, a tensile strength of $448 \mathrm{MPa}$; and for 19-mm-thick tracks, a tensile strength of $345 \mathrm{MPa}$. Table 2 presents the comparison of the ultimate tested $\left(P_{\max }\right)$ and the estimated capacity as well as the ratio of these values. The equations predicted the tested capacity very well. Note that for design purposes, the AISI S100-12 [25] reduces the calculated capacity by factors of 1.7 and 2.8 ( $\Omega$ factors) for web-crippling and deflection-track damage states, respectively.

\subsection{Effect of Loading Rate}

In order to evaluate the effect of loading rate on the experimental results, the monotonic test of the first series of specimens was repeated with three different loading rates $(0.04$ $\mathrm{mm} / \mathrm{sec}, 0.21 \mathrm{~mm} / \mathrm{sec}$, and $0.42 \mathrm{~mm} / \mathrm{sec}$ ). Fig. $6 \mathrm{a}$ and Fig. $6 \mathrm{~b}$ compare the force-displacement responses and maximum force ratios in these monotonic tests. The maximum force ratio was defined as the ratio of the maximum force of each monotonic test to the maximum force of the monotonic test with a loading rate of $0.04 \mathrm{~mm} / \mathrm{sec}$. The comparison demonstrates a negligible variation in test results with an unclear trend. Moreover, observed damage mechanisms were similar in these three tests. Accordingly, the performance of stud-to-track 
connections in the out-of-plane direction was assumed to be insensitive to the loading rate and a constant loading rate of $0.21 \mathrm{~mm} / \mathrm{sec}$ was used for the rest of the experiments.

\subsection{Force-displacement response}

The backbone curves of the cyclic tests, the median of the backbone curves, and the monotonic response of specimen series T48G03DT and T75G13DT, as two examples of tested series, are displayed in Fig. 7. The discrepancies between the responses of the three specimens in each series were marginal. The monotonic and cyclic responses were analogous in terms of initial stiffness, maximum force capacity, and observed damage mechanisms. Nonetheless, the complete failure of connections was usually initiated in lower displacements during the cyclic tests in comparison with the monotonic tests. This difference was mainly due to the effect of cumulative damage on the cyclic responses.

Fig. 8a discloses the effect of stud-to-track gaps on the connection response. The figure indicates that a smaller gap leads to higher initial stiffness, larger force capacity, and larger failure displacement (the displacement associated with the complete failure of the connection). Furthermore, using a larger gap can change the dominant damage mechanism from track-flange deflection $(E D)$ to stud-web crippling $(W C)$ (see Table 2). The responses of connections with $48-\mathrm{mm}$ - and $75-\mathrm{mm}$ - thick studs/tracks, as well as connections with deflection-track and screw-attached configurations are compared in Fig. 8b. As expected, the initial stiffness and maximum force capacity were larger in connections with thicker studs/tracks and screw attachment. The reason is that the force characteristics of the specimen responses were controlled by the mechanisms (either $W C$ or $E D$ ) that were enhanced by increasing stud and track thicknesses. However, utilizing thicker profiles limited the stud and track deformation. In fact, the thicker specimens usually failed at smaller displacement 
compared to the thinner specimens. Screwing the studs to the tracks also introduced additional stiffness and strength to the connection. The failure displacements of screwattached connections were commonly larger than deflection-track connections.

\section{Capacity fragility analysis}

Fragility curves state the probability of experiencing or exceeding a specific damage state $(D S)$ conditioned on a particular value of an engineering demand parameter $(E D P)$. The required steps to generate the fragility curves include: 1) choose a proper fragility formulation, 2) select appropriate engineering demand parameters, 3) determine capacity (damage state) estimates, and 4) develop fragility curves [26].

A number of methodologies for generating capacity fragility curves have been developed in the past. In this study, method $A$ proposed by Porter et al. [27] was adopted to assess the vulnerability of stud-to-track connections in the out-of-plane direction. This method can be used in experimental studies in which all specimens reach all DSs at observed values of EDPs. This is most common where the damage can be associated with a point on the observed force-displacement of a component [27]. Porter et al. utilized a lognormal probability distribution, $F_{d m}(e d p)$, to define the probability that the component reaches or exceeds damage state $d m$, given a particular $E D P$ value, $P[D M \geq d m \mid E D P=e d p]$, as follows:

$$
F_{d m}(e d p) \equiv P[D M \geq d m \mid E D P=e d p]=\Phi\left(\frac{\ln \left(e d p / x_{m}\right)}{\beta}\right)
$$

where $\Phi$ denotes the standard normal (Gaussian) cumulative distribution function, $x_{m}$ indicates the median value of the distribution, and $\beta$ represents the logarithmic standard 
deviation [27]. The EDPs should be chosen to be most closely related to the failure probability of the specimen. The cyclic performance of the stud-to-track connections were mainly governed by the displacement of the stud. Therefore, this displacement was considered as the main EDP. However, the connection force was also used as an alternate demand parameter.

Three damage states were defined for stud-to-track connections based on the extent of the nonlinearity in connections and observed damage mechanisms. The first damage state (DS1), which represented the initiation of nonlinearity in the connection, was defined as the displacement associated with $0.40 P_{\max }$ [28]. This point correlated with the observation of a slight deformation in track flanges $(S D)$ and can be considered as the serviceability limit. The second damage state (DS2) was set to the local maximum point on the backbone curve. Excessive track-flange deflection $(E D)$ and/or stud-web crippling $(W C)$ were observed at this damage state. Note that in addition to the more common issues related to excessive deformation such as cracking of finishes, large deformation at the stud-to-track location could prevent the proper operation of windows and doors. In this case, excessive deflection would be a limit state on safety rather than serviceability [22]. At the third damage state $(D S 3)$, studs were permanently displaced $(P D)$ and pushed out of tracks. Fig. 9 depicts the $D S s$ with their associated points on two representative backbone curves.

In method $A$, the individual damage states are characterized by representative values for the median, $x_{m}$, and dispersion, $\beta$, for the component damage state distributions as follows (Porter et al. 2007, Soroushian et al. 2014):

$$
x_{m}=e^{\frac{1}{N} \sum_{i=1}^{N} \ln \left(x_{i}\right)}
$$




$$
\beta=\sqrt{\frac{1}{N-1} \sum_{i=1}^{N}\left[\ln \left(\frac{x_{i}}{x_{m}}\right)\right]^{2}}
$$

where $x_{i}$ denotes the i-th measured displacement corresponding to specific damage observation (EDPs) and $N$ is the number of cyclic tests conducted for each group of specimens. The fragility curves were generated for each series of specimens; therefore, $N$ was equal to 3 for all groups. Table 3 and Table 4 summarize the EDPs $\left(x_{i}\right), x_{m}$, and logarithmic standard deviation obtained for each series and damage level.

Fig. 10a, Fig. 10b, and Fig. 10c depict the fragility curves for stud-to-track connections in the out-of-plane direction, considering displacement as EDP. A smaller stud-to-track gap improves the connection in the first and last damage states (DS1 and DS3) but may deteriorate the connection performance in DS2. The curves also indicate that the connections with thicker studs/tracks are more vulnerable in all damage states. There was no clear difference between the deflection-track and screw-attached configurations in the first two damage states. However, the screw attachment could postpone the complete failure of the connection (DS3). A similar trend can be found by comparing the median values $\left(x_{m}\right)$ in Table 4. It should be mentioned that the Lilliefors goodness-of-fit test at 5\% significance level [29] was performed for each series of specimens in order to check the validity of the lognormal distribution. The test was satisfied, therefore the lognormal distribution appropriately fitted the data.

An alternative set of fragility curves (for $D S 2$ ) based on the connection force is presented in Fig. 10d. The figure reveals that although smaller gaps, thicker profiles, and screw attachment might increase the probability of occurrence of $D S 2$ in terms of connection 
displacement, they augment the connection in terms of force capacity. The fragility curves of Fig. 10d can be adopted, in lieu of the fragility curves of Fig. 10b, where the force-based design methodology is desired rather than the displacement-based. Consider that Fig. 10d can be converted to the fragility curves of $D S 1$ by scaling the horizontal axis by a factor of 0.4 .

\section{Development of a numerical hysteresis model for stud-to-track connections in out-of-plane direction}

The experimental data was employed to develop a numerical hinge material model for the behavior of stud-to-track connections in the out-of-plane direction. A one-dimensional hysteresis load-displacement relationship is defined using the "Pinching4" uniaxial material along with a "zeroLength" element in OpenSees [30]. This material enables the simulation of complex, pinched force hysteresis responses accounting for degradations under cyclic loadings [26] similar to one shown in Fig. 5(b). The "Pinching4" material model requires the definition of 39 parameters as presented in Fig. 11. Sixteen parameters describe the backbone curve in positive (ePdi and $e P f i$ ) and negative directions (eNdi and $e N f i)$, while an additional eight parameters characterize the "pinched" or unloading/reloading behavior of the model. The pinching parameters include the ratio of reloading/maximum historic deformation $r \operatorname{Disp}(P-N)$, the ratio of reloading/maximum historic force $r F o r c e(P-N)$, and the ratio of negative (positive) unloading/maximum (minimum) monotonic strength $u$ ForceP(N) [26].

Unloading and reloading stiffness degradation as well as strength degradation can be considered in the model using $g K i, g D i$, and $g F i$. A detailed description of these parameters can be found at the OpenSees website [30]. 


\subsection{Calibration of proposed numerical model using experimental data}

For each specimen, a "Pinching4" material was calibrated so that the hysteresis response, the value of cumulative hysteresis energy, and the force histories fit the experimental data on a visual basis [26]. In addition, the error in the maximum cumulative hysteresis energy was checked to be less than $10 \%$. The recorded displacement histories were utilized as the inputs for numerical analysis. Fig. 12 describes the calibration process for one sample stud-to-track connection from the T48G03DT series.

Initially for each specimen, all 39 parameters of "Pinching4" material were calibrated to find the best correlation between numerical results and experimental data. However, after performing a sensitivity analysis on the parameters, it was noted that the force and stiffness degradation parameters $(g K i, g D i$, and $g F i)$ as well as the pinching and unloading/reloading parameters (23 out of 39 parameters) could be fixed. Therefore, constant values were assigned to these parameters (Table 5). Subsequently, to generate a numerical model with a backbone curve consistent with the experimental results, backbone points were determined for each particular specimen. Table 6 presents examples of the values used to define the backbone curves. Fig. 13 displays the comparisons of numerical and experimental results for two sample specimens.

\subsection{Development of generic models for tested connections}

In the previous section, a total of 18 sets of the 16 backbone parameters of "Pinching4" material $\left(e P d_{i}, e P f_{i}, e t c\right)$ were optimized. A comparison of these sets indicated that there were major variations between the backbone parameters of specimens of different series, while the discrepancies between the backbone parameters of the three specimens within each series were minimal. In order to facilitate the future numerical modeling of stud-to-track 
connections, one suite of material parameters was defined as the generic parameters for every specimen series, called the generic model. These generic models represent the stud-to-track connections with properties (stud and track thickness, gap size, and connection configuration) similar to what were tested in each series. Inspired by the work of Soroushian et al. [26], the generic models were developed following these assumptions: 1) the displacement points of the backbone curve (in each direction), $e P d_{1}, e P d_{2}, e P d_{3}, e P d_{4}$ (Fig. 11), are set to the median of the calibrated values corresponding to each of these points of the backbone curve; 2) a linear interpolation is used to find the force corresponding to the previously mentioned displacements where the force values at the calibrated backbone curves are unavailable. The median of these force values for each set denotes the backbone points in each direction $\left(e P f_{l}\right.$ $e P f_{2}, e P f_{3}$, and $e P f_{4}$ in Fig. 11); 3) the remainder of the parameters (fixed parameters) are the same as those provided in Table 5.

The generic model parameters, obtained based on the aforementioned assumptions, are presented in Table 7. As an example, Fig. 14a shows the comparison between the generic backbone curves and all the calibrated backbone curves of series T75G50AS. Fig. 14b compares the hysteresis response of this generic model to the experimental data of specimen \#2.

\subsection{Proposed generic models for untested stud-to-track connections}

The generic models for stud-to-track connections with three specific gaps and two stud and track thicknesses were provided in the preceding section. However, the connection gap and/or thickness in practical works might be different from the tested values. Therefore, an approximate procedure is proposed in this section to generate numerical models of stud-totrack connections for any thicknesses and/or gaps. The proposed method is based on the 
assumption that the maximum connection capacity is governed by either track-flange deflection or stud-web crippling. Supplemental experiments with a wider range of stud and track profiles, material properties, gaps, and screw types are essential in order to enhance the proposed model in the future.

A normalized generic backbone curve was developed for stud-to-track connections with deflection-track configuration using the following procedure: 1) the backbone curve is considered to be symmetric; 2$)$ the first backbone-point displacement $\left(e P d_{l}\right)$ was assumed to be constant (independent of the connection properties) and equal to $0.2 \mathrm{~mm}$; 3) the secant stiffness was found for the last three backbone points of each specimen (Fig. 15a); 4) the secant stiffness values were plotted against the corresponding values of $\mathrm{t} \times \mathrm{b}_{\text {eff }} / \mathrm{e}$, where $e$ is the gap dimension, $t$ represents the stud (and track) thickness, and $b_{\text {eff }}$ denotes the effective flange length, defined by Eqs. (5); 5) the least-square regression method is utilized to fit a line to the data (Fig. 16); 6) the equations of these lines can be used to determine the secant stiffness of backbone curves of stud-to-track connections with gap and/or profile thickness other than those that were tested in this study; 7) all the backbone curve force values (ePf and $e N f_{i}$ ) of each specimen model are normalized with respect to the maximum force value $\left(e P f_{3}\right)$ (Fig. 15b); 8) the median of nine normalized force values corresponding to each backbone point are used to define the normalized generic backbone curve (Table 8). The normalized generic backbone curve (Fig. 17a) can be multiplied by the ultimate connection capacity (the lesser of the values calculated from Eqs. (1) and (4)) to find force values of the numerical backbone curve for connections with any stud and track thicknesses and/or gap size. Afterwards, the force values can be divide by the secant stiffness values (calculated based on the fitted line in Fig. 16) to find the displacement of the backbone points. 
Screwing the stud to the tracks introduces additional strength to the connection that mainly affects the force values of the second and third backbone points $\left(e P f_{2}, e P f_{3}, e P N_{2}\right.$, and $e N f_{3}$ ) (see Table 7). Thus, for screw-attached configurations (with gap dimensions and/or profile thicknesses other than those tested in this study), the numerical backbone curve can be generated following these steps: 1) an initial backbone curve is developed assuming a trackdeflection configuration and based on Fig. 17a, 2) the force values of second and third backbone points are increased as much as $R \times P_{\text {not }}$ (see Eq. (3)). A schematic development of a backbone curve for a screw-attached connection is illustrated in Fig. 17b.

\section{Summary and Conclusions}

Cold formed steel stud-to-track connections were tested under monotonic and reverse cyclic loading as part of a larger investigation of nonstructural partition wall behavior. Stud and track thickness, stud-to-track gap, and the connection configurations (either screwattached or deflection-track) were varied between specimens. The test program was designed to assess the out-of-plane displacement and strength capacities and stiffness of stud-to-track connections in nonstructural partition walls.

The main observations and conclusions obtained from the experimental study are as follows:

- The dominant damage mechanism was excessive track-flange deformation for connections with large stud-to-track gap (larger than $3 \mathrm{~mm}$ in this study) and stud-web crippling for other connections. Where studs were screwed to tracks, the screws were pulled out from the studs after the web crippling. 
- The equations proposed by Bolte and LaBoube [19] and AISI S100-12 [25] could appropriately estimate the ultimate force capacity of the connection.

- Increasing the stud-to-track gap not only affects the dominant damage mechanism, but also leads to lower initial stiffness, smaller force capacity, and smaller failure displacement. Moreover, the initial stiffness and maximum force capacity were larger in connections with thicker studs/tracks and attached screws. The connections with thicker studs/tracks were less ductile compared to the thinner connections.

- The displacement-based fragility analysis revealed that: 1) a smaller stud-to-track gap improves the connection in the first and last damage states (DS1 and DS3) but may deteriorate the connection performance in $D S 2,2)$ connections with thicker studs/tracks are more vulnerable in all damage states, 3) adding screws can postpone the complete failure of the connection (DS3).

- The experimental data was employed to generate and calibrate a series of nonlinear hinge models for all specimens. Subsequently, for each series a generic model was defined that represented a stud-to-track connection with properties (stud and track material and thickness, stud-to-track gap, and screw type) similar to those that have been tested in that series. In addition, an approximate procedure is proposed to generate numerical models of stud-to-track connections with any profile thicknesses and/or gap dimensions.

The hinge model of stud-to-track connections could be utilized along with the numerical models of other wall components (such as gypsum-to-stud connections and steel studs) to numerically model the in-plane and out-of-plane behavior of a partition wall assembly [31]. The model could be subjected to realistic input motions (e.g. floor accelerations) to estimate 
the demand parameters on each component. These demand estimations could be used in conjunction with the capacity parameters (e.g. median and deviation) developed in this study (and similar studies for other partition wall components) to generate fragility curves for partition wall systems in terms of more global engineering demand parameters, such as floor accelerations and/or inter-story drifts

\section{Acknowledgments}

The current material is based upon work supported by the National Science Foundation under Grant No. 0721399. This Grand Challenge (GC) project to study the seismic response of nonstructural systems is under the direction of $\mathrm{M}$. Maragakis from the University of Nevada, Reno and Co-PIs: T. Hutchinson (UCSD), A. Filiatrault (UB), S. French (G. Tech), and B. Reitherman (CUREE). Any opinions, findings, conclusions, or recommendations expressed in the current document are those of the investigators and do not necessarily reflect the views of the sponsors. The input provided by the Practice Committee of the NEES Nonstructural Project, composed of W. Holmes (Chair), D. Allen, D. Alvarez, and R. Fleming, and by the Advisory Board, composed of R. Bachman (Chair), S. Eder, R. Kirchner, E. Miranda, W. Petak, S. Rose and C. Tokas, has been crucial for the completion of this research. Assistance from M. Lattin of the University of Nevada, Reno material lab during the assembly and testing as well as support from Johnnie Stolz of Omboli Interior Inc. is appreciated.

\section{Nomenclature}

T48 or T75 connections with $0.48-$ or $0.75-\mathrm{mm}$ thick studs and tracks, respectively 
G03, G13, or G22 connections with 3, 13, or $22 \mathrm{~mm}$ gap, respectively

SA connections with the screw-attached configuration

DT connections with the deflection-track configuration

SD a damage mechanism defined as the slight track-flange deformation

ED a damage mechanism defined as the excessive track-flange

deformation

PD a damage mechanism defined as the permanent displacement of studs

WC a damage mechanism defined as the crippling of the stud web

SP a damage mechanism defined as the pulling out of the screw from stud

\section{References}

1. Taghavi S, and Miranda E. Response Assessment of Nonstructural Building Elements, PEER Report 2003/05, Pacific Earthquake Engineering Research Center (PEER), Berkeley, CA, 2003.

2. Restrepo JI, Lang AF. Study of Loading Protocol in Light-Gauge Stud Partition Wall. Earthquake Spectra 2011; 27:1169-1185. DOI: 10.1193/1.3651608.

3. Dhakal RP. Damage to non-structural components and contents in 2010 Darfield earthquake. Bulletin of The New Zealand Society for Earthquake Engineering 2010; 43:404-411.

4. Baird A, Tasligedik AS, Palermo S, Pampanin S. Seismic performance of vertical non-structural components in the $22^{\text {nd }}$ February 2011 Christchurch earthquake. Earthquake Spectra 2014; 30(1), 401-425. DOI: 10.1193/031013EQS067M.

5. Mizutani K, Kim H, Kikuchihara M, Nakai T, Nishino M, Sunouchi S. The damage of the building equipment under the 2011 Tohoku pacific earthquake. 9th International Conference on Urban Earthquake Engineering \& 4th Asia Conference on Earthquake Engineering, Tokyo Institute of Technology, Tokyo, Japan, 2012.

6. EERI (Earthquake Engineering Research Institute). The El Mayor Cucapah, Baja California earthquake April 4, 2010. An EERI Reconnaissance Rep 2012; 2010-02, J. Meneses, ed., Oakland, CA.

7. Miranda E, Mosqueda G, Retamales R, Pekcan G. Performance of Nonstructural Components during the 27 February 2010 Chile Earthquake. Earthquake Spectra 2012; 28:S1:S453-S471. DOI: $10.1193 / 1.4000032$.

8. Wood RL, Hutchinson TC. Design-Oriented Model for Capturing the In-Plane Seismic Response of Partition Walls. ASCE Journal of Structural Engineering 2014, 140. DOI: 
10.1061/(ASCE)ST.1943-541X .0000899.

9. Wang X, Pantoli E, Hutchinson T, Restrepo J, Wood R, Hoehler M, Grzesik P, and Sesma F. Seismic Performance of Cold-Formed Steel Wall Systems in a Full-Scale Building, 2015, ASCE Journal of Structural Engineering. DOI: 10.1061/(ASCE)ST.1943-541X.0001245.

10. Fülöp LA, Dubina D. Performance of Wall-Stud Cold-Formed Shear Panels under Monotonic and Cyclic Loading, Part I: Experimental research. Thin-Walled Structures 2004; 42: 321-338. DOI: 0.1016/S0263-8231(03)00063-6.

11. Lee T, Kato M, Matsumiya T, Suita K, Nakashima M. Seismic Performance Evaluation of Nonstructural Components: Drywall Partitions. Earthquake Engineering and Structural Dynamics 2007; 36: 367-382. DOI: 10.1002/eqe.638.

12. Retamales R, Mosqueda G, Filiatrault A, Reinhorn AM. New Experimental Capabilities and Loading Protocols for Seismic Qualification and Fragility Assessment of Nonstructural Components. Technical Report MCEER-08-0026, State University of New York at Buffalo, NY, 2008.

13. Restrepo JI, Bersofsky A. Performance Characteristics of Light Gage Steel Stud Partition Walls. Thin-Walled Structures 2010; 49:317-324. DOI: 10.1016/j.tws.2010.10.001.

14. Soroushian S, Ryan KL, Maragakis M, Wieser J, Sasaki T, Sato E, Okazaki T, Tedesco L, Zaghi AE, Mosqueda G, Alarez D. NEES/E-Defense tests: Seismic performance of ceiling/sprinkler piping nonstructural systems in base isolated and fixed base building. 15th World Conference on Earthquake Engineering (15WCEE), Lisbon, Portugal, 2012.

15. Retamales R, Davies R, Mosqueda G, Filiatrault A. Experimental Seismic Fragility of ColdFormed Steel Framed Gypsum Partition Walls. ASCE Journal of Structural Engineering 2013; 139:1285-1293. DOI: 10.1061/(ASCE)ST.1943-541X.0000657.

16. Rahmanishamsi E, Soroushian S, Maragakis M. System-Level Experiments on Ceiling/Piping/Partition Systems at UNR-NEES Site. Tenth U.S. National Conference on Earthquake Engineering, Anchorage, AK, 2014. DOI: 10.4231/D3BR8MG8F.

17. Jenkins, C., Soroushian, S., Rahmanishamsi, E., and Maragakis, M. Experimental Fragility Analysis of Cold-Formed Steel-Framed Partition Wall Systems, Structural Congress, ASCE, Portland, OR, 2015. DOI: 10.1061/9780784479117.152.

18. Fox SR., Schuster RM. Lateral strength of wind load bearing wall stud-to-track connection. Proceedings of the 15th International Specialty Conference on Cold-Formed Steel Structures, Rolla (MO): University of Missouri -Rolla, 2000.

19. Bolte, W.G., LaBoube, R.A. Behavior of Curtain Wall Stud to Track Connections, Thin-Walled Structures 2004, 42, 1431-1443, DOI: 10.1016/j.tws.2004.01.005.

20. North American Specification for the Design of Cold- Formed Steel Structural Members. American Iron and Steel Institute, Washington, DC, 2001.

21. United States Army Corps of Engineers. Design of cold-formed load-bearing steel systems and masonry veneer/steel stud wall. Technical Instructions TI 809-07, Washington, DC; 1998.

22. Lewis, A.V., Fox, S.R., Schuster, R.M. Strength of Cold-Formed Steel Jamb Stud-to-Track Connections, Nineteenth International Specialty Conference on Cold-Formed Steel Structures, St. Louis, Missouri, 2008.

23. Rahmanishamsi E, Soroushian S, Maragakis E. M. Cyclic Shear Behavior of Gypsum Board-toSteel Stud Screw Connections in Nonstructural Walls. Earthquake Spectra 2015. In-Press. DOI: 10.1193/062714EQS091M 
24. Retamales, R., Mosqueda, G., Filiatrault, A., and Reinhorn, A.M. Testing protocol for experimental seismic qualification of distributed nonstructural systems. Earthquake Spectra 2011, 27(3):835-856, DOI: 10.1193/1.3609868.

25. AISI S100-12, North American Specification for the Design of Cold- Formed Steel Structural Members. American Iron and Steel Institute, Washington, DC, 2012.

26. Soroushian S, Zaghi AE, Maragakis M, Tian T, Filiatrault A. Analytical Seismic Fragility Analyses of Fire Sprinkler Piping Systems with Threaded Joints. Earthquake Spectra 2015, 31, 1125-1155. DOI: 10.1193/083112EQS277M.

27. Porter, K., R. Kennedy and R. Bachman. Creating Fragility Functions for Performance-Based Earthquake Engineering. Earthquake Spectra 2007, 23, 471-489, DOI: 10.1193/1.2720892.

28. Peterman, K.D., Nakata, N., and Schafer, B.W. Hysteretic Characterization of Cold-formed Steel Stud-to-Sheathing Connections. Journal of Construction Steel Research 2014. 101, 254-264, DOI: $10.1016 /$ j.jcsr.2014.05.019.

29. Lilliefors, H. On the Kolmogorov-Smirnov test for normality with mean and variance unknown. Journal of the American Statistical Association 1967, 62, 399-402, DOI: 10.2307/2283970.

30. Open System for Earthquake Engineering Simulation (OpenSees) website. http://www.opensees.berkeley.edu . PEER, Berkeley, CA, 2015.

31. Rahmanishamsi E, Soroushian S, Maragakis E. M. Analytical model for the in-plane seismic performance of cold-formed steel-framed gypsum partition walls. Earthquake Engineering and Structural Dynamics 2015; DOI: 10.1002/eqe.2676. 


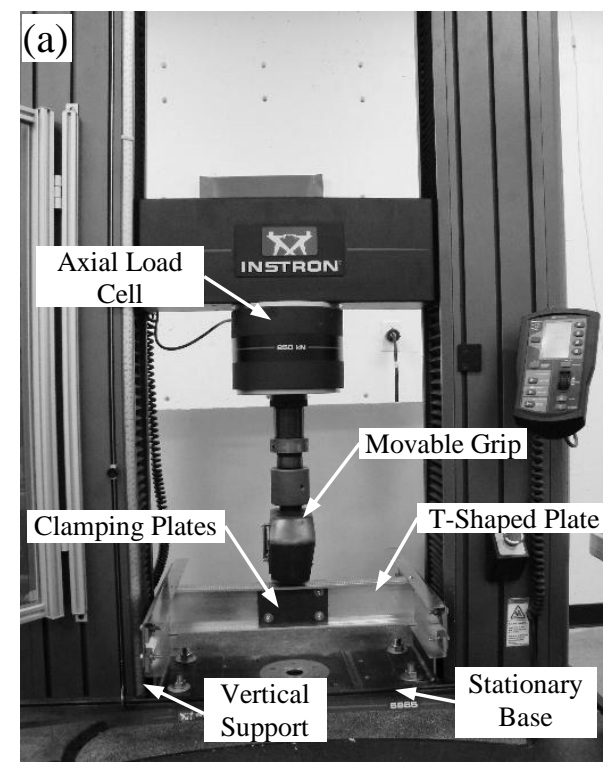

(b)

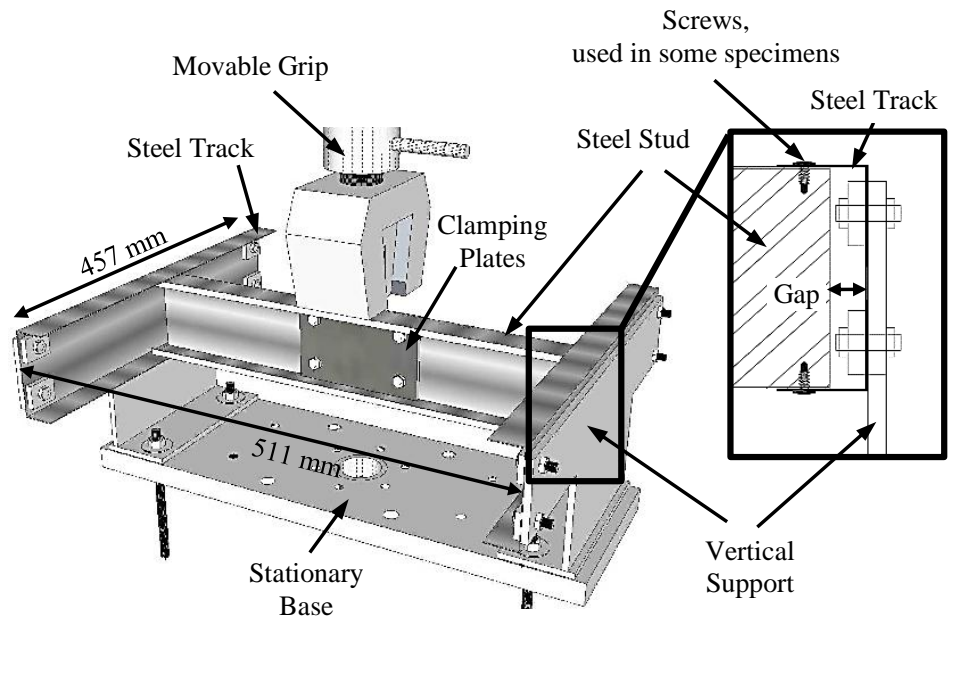

Fig. 1. (a) and (b) Specimen and Test Machine 


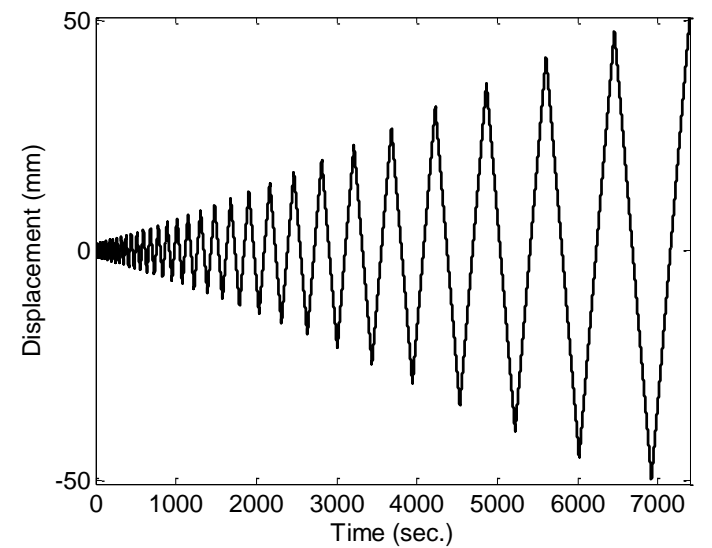

Fig. 2. Loading protocol for cyclic tests 


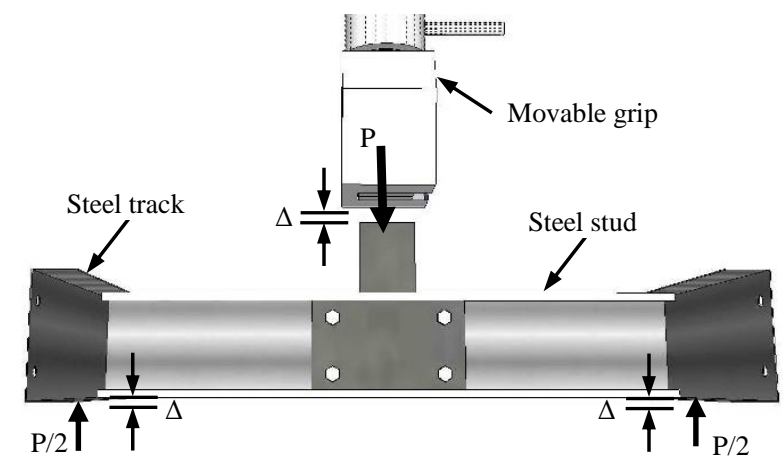

Fig. 3. Free-body diagram for a sample specimen 


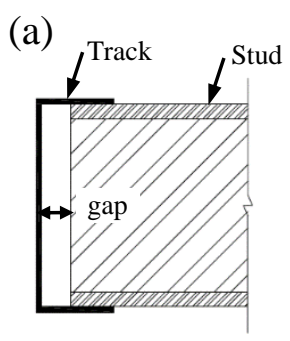

(b)
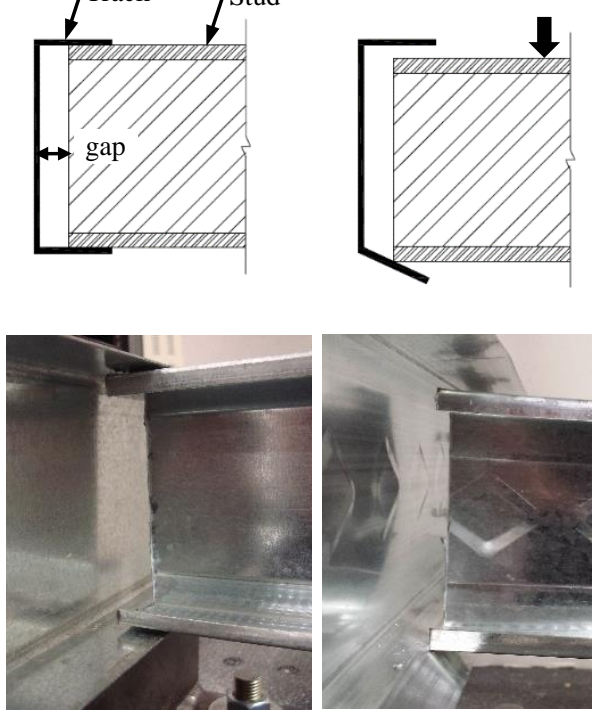

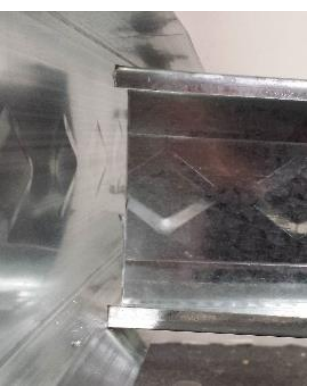

(c)

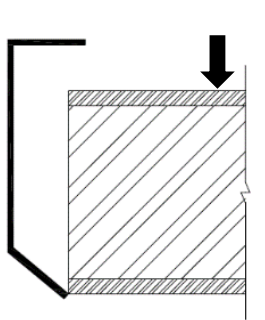

(d)
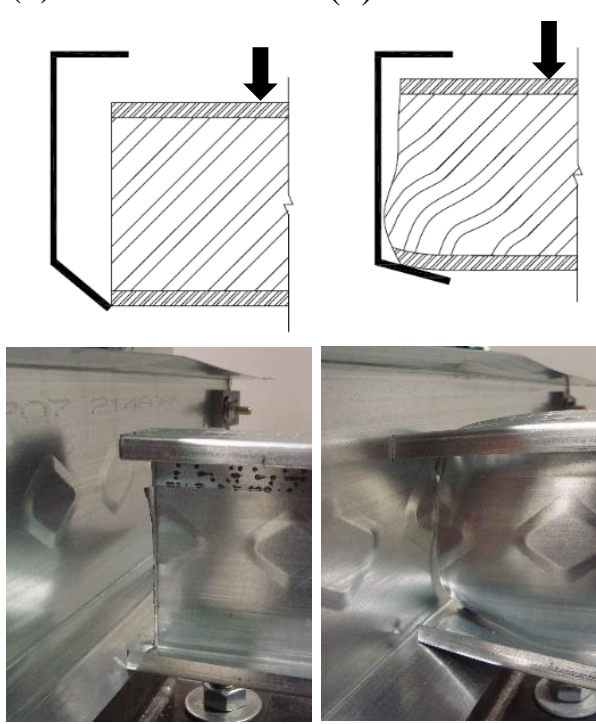

(e)
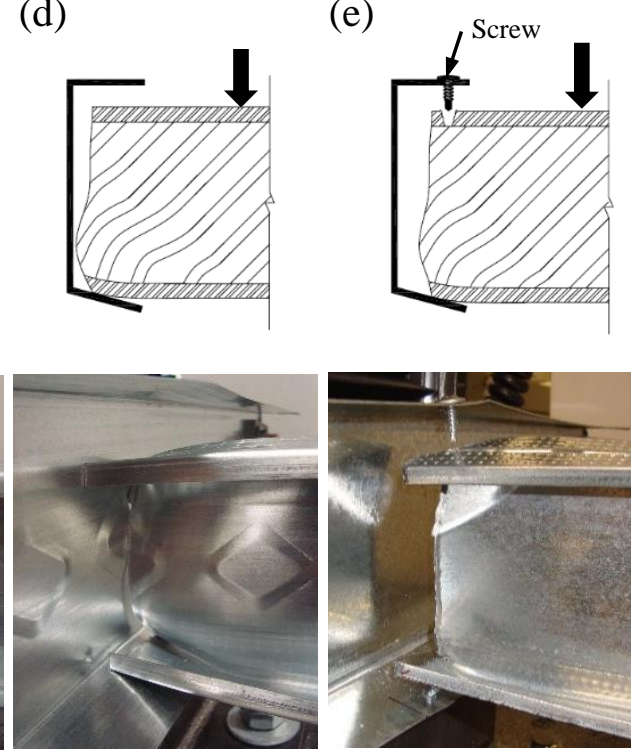

Fig. 4. Damage mechanisms of stud-to-track connections in the out-of-plane direction: (a) initial condition, (b) track-flange deformation, (c) permanent displacement of the stud, (d) stud-web crippling, (e) screw pull-out 

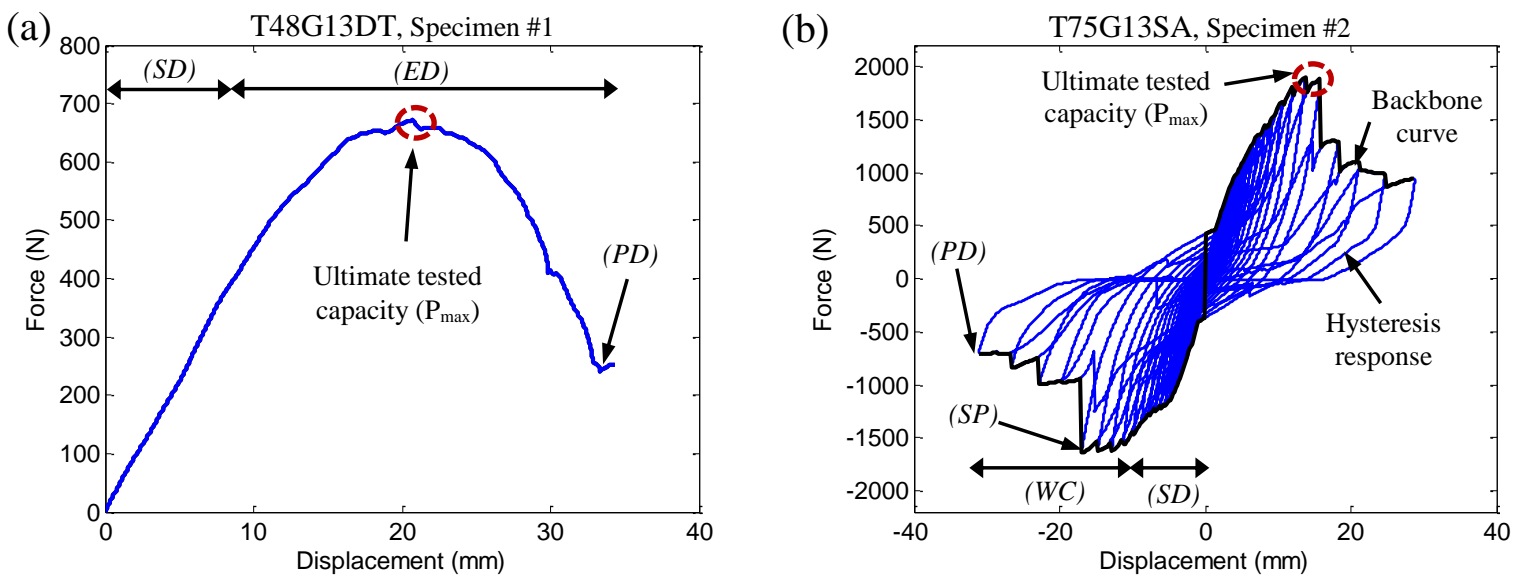

Fig. 5. Typical experimental responses for (a) monotonic and (b) cyclic tests 
(a)

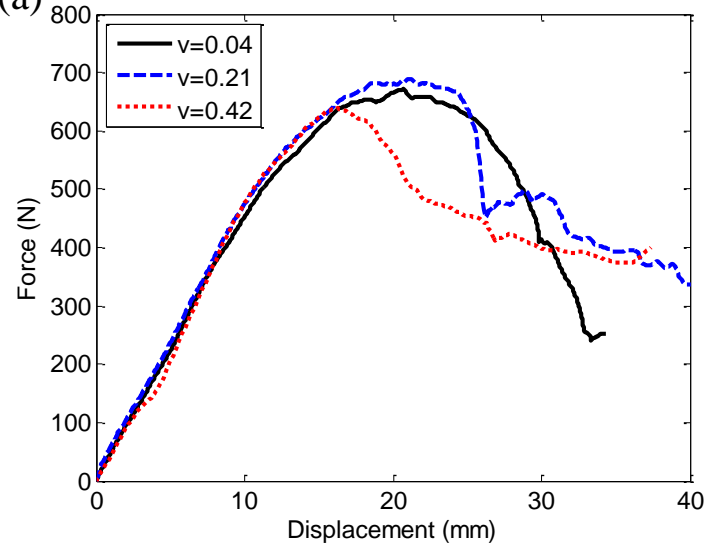

(b)

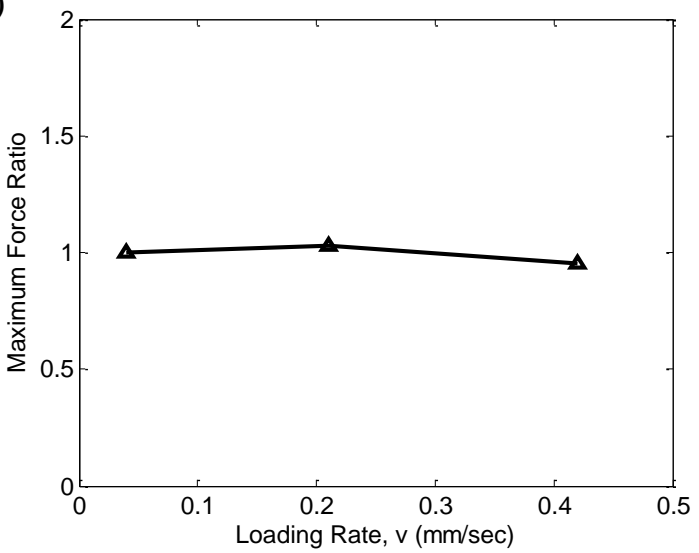

Fig. 6. Effect of loading rate (v, $\mathrm{mm} / \mathrm{sec})$ on: (a) monotonic force-displacement response, (b) maximum force ratio 



Fig. 7. Monotonic response and cyclic backbone curves for specimen series: (a) T48G03DT and (b) T75G13AS 
(a)



(b)

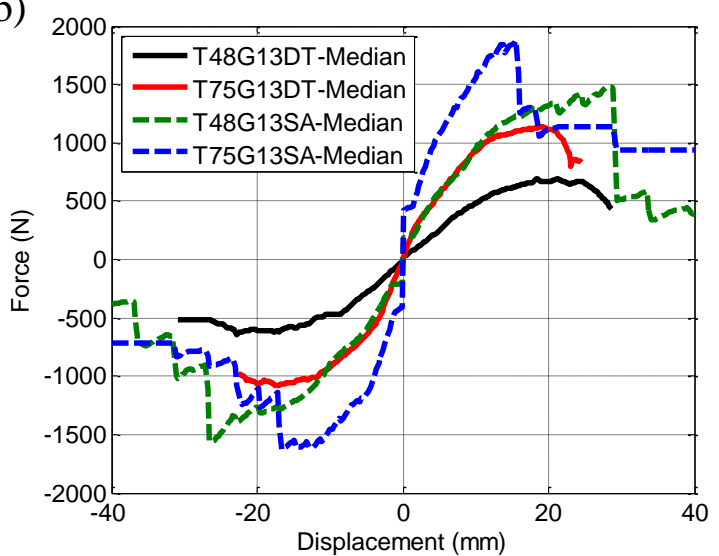

Fig. 8. Effect of (a) stud-to-track gap, (b) stud/track thickness and screw-attached configuration on the cyclic response 

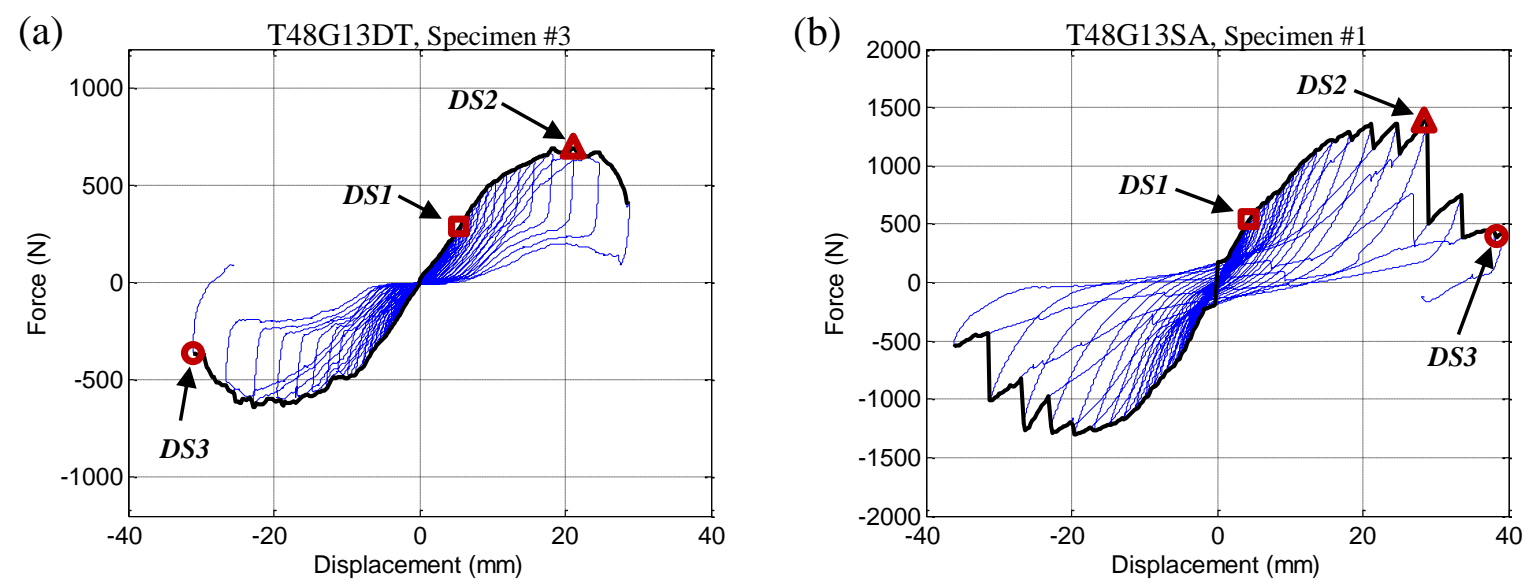

Fig. 9. Examples of damage state definitions for: (a) deflection-track configuration (b) screw-attached configuration 
(a)
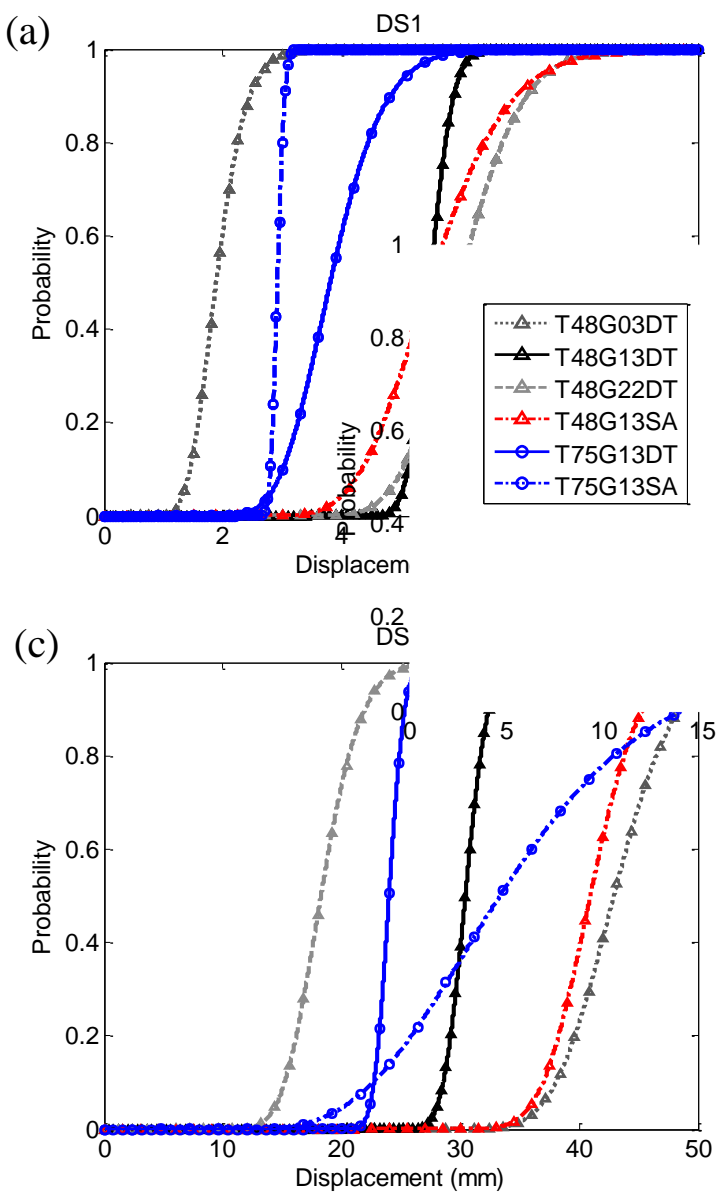

(b)
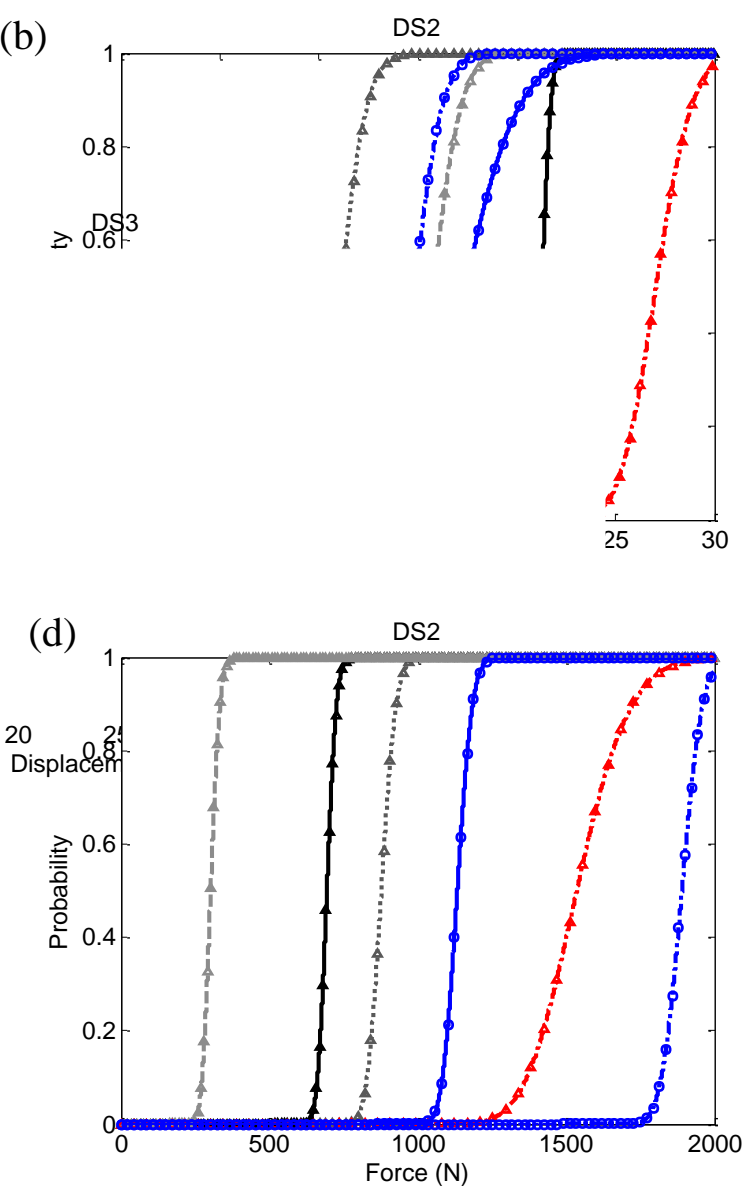

Fig. 10. Displacement-based fragility curves for (a) first damage state, (b) second damage state, (c) third damage state, and (d) force-based fragility curves for second damage state 


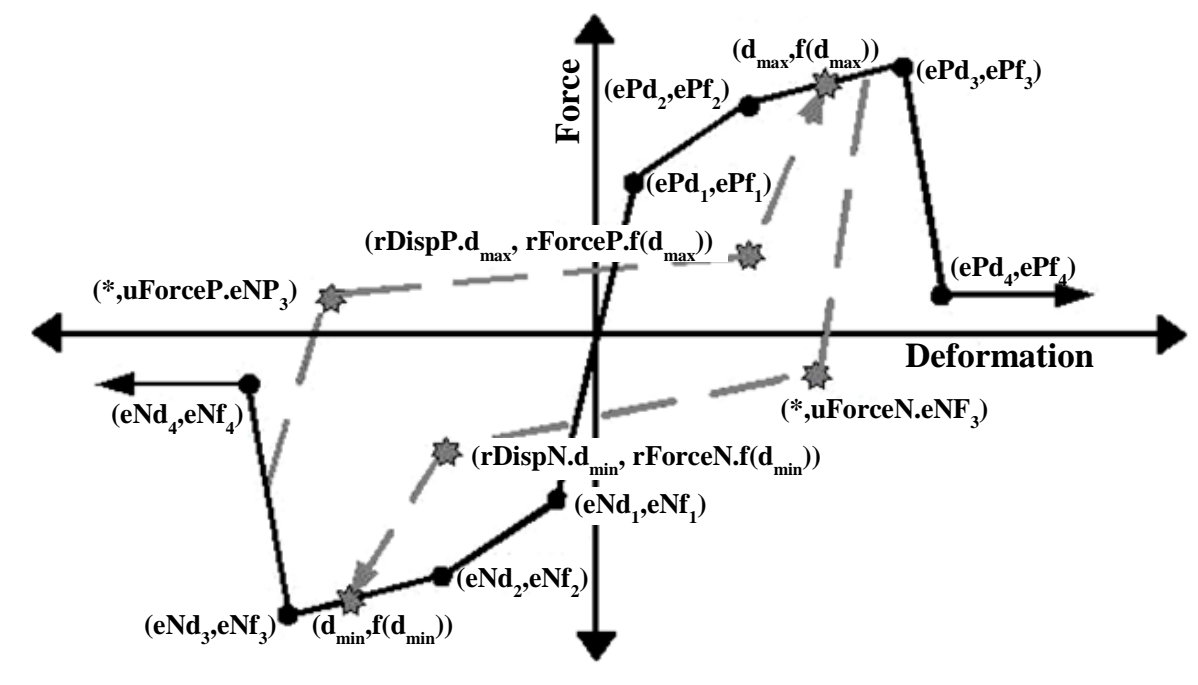

Fig. 11. Pinching 4 material properties (OpenSees 2015) 

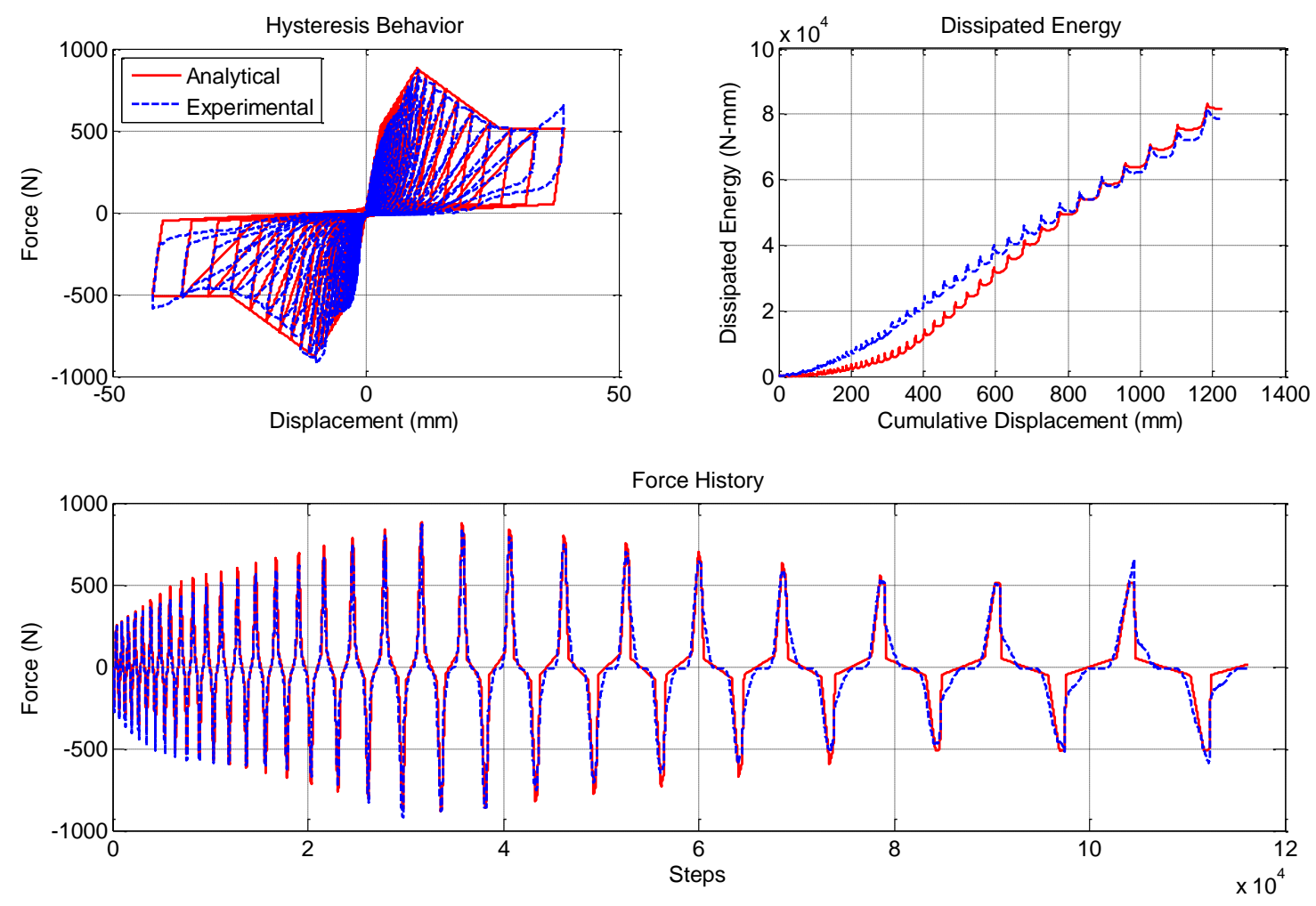

Fig. 12. Calibrated numerical model for a sample specimen (T48G03DT, specimen \#2) 



Fig. 13. Sample numerical-experimental hysteresis comparisons of specimen series (a) T48G13AS

(b) T75G13DT 

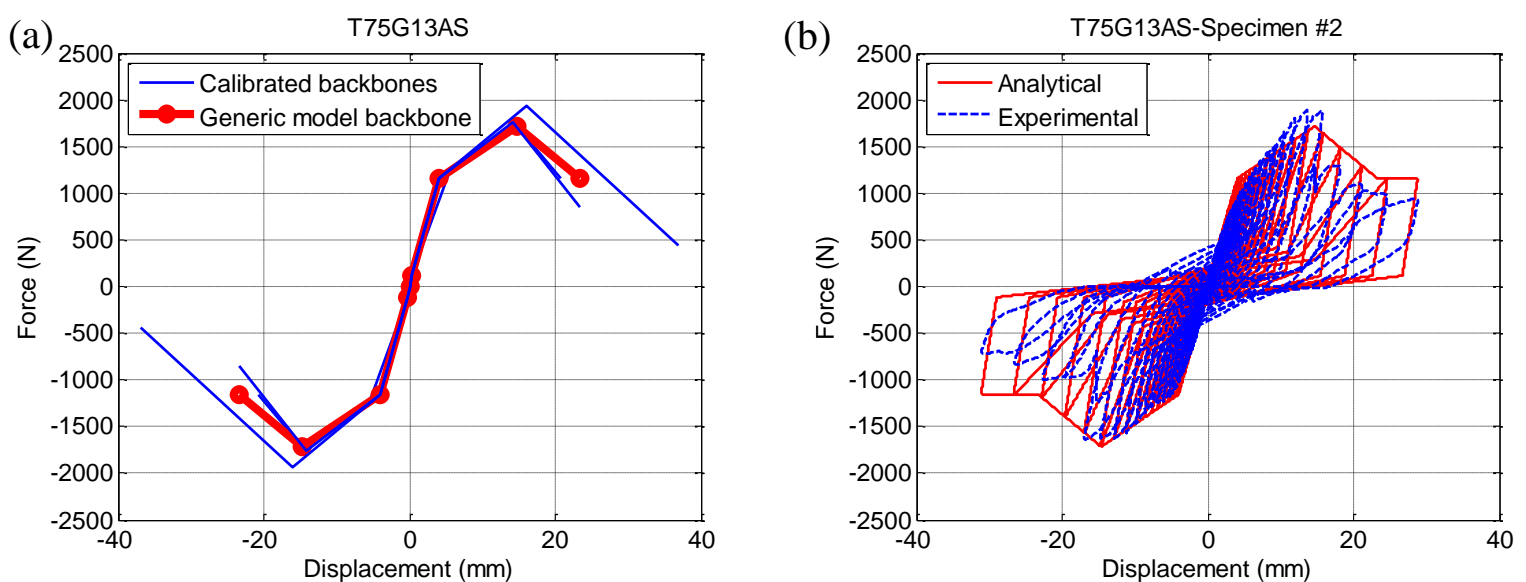

Fig. 14. (a) Generic backbone curves of specimen series T75G13AS, (b) Sample numericalexperimental hysteresis comparison 

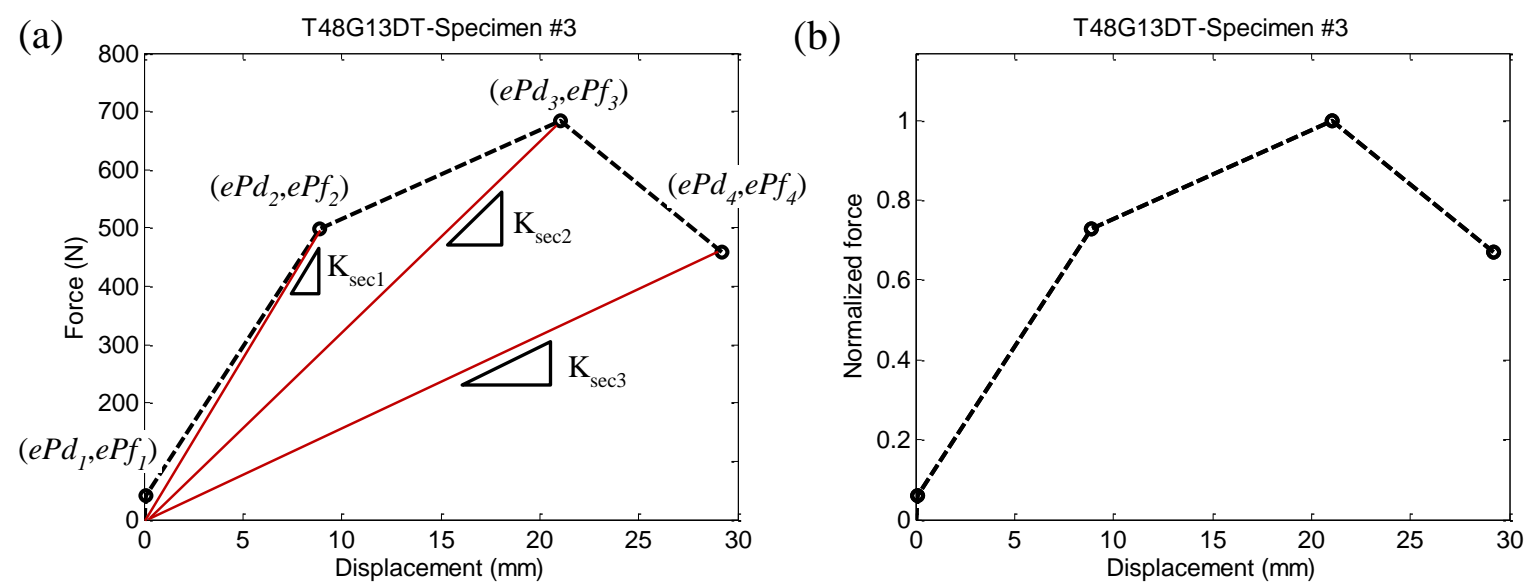

Fig. 15. (a) Secant stiffness calculation on a sample specimens, (b) force normalized backbone curve of a sample specimen 

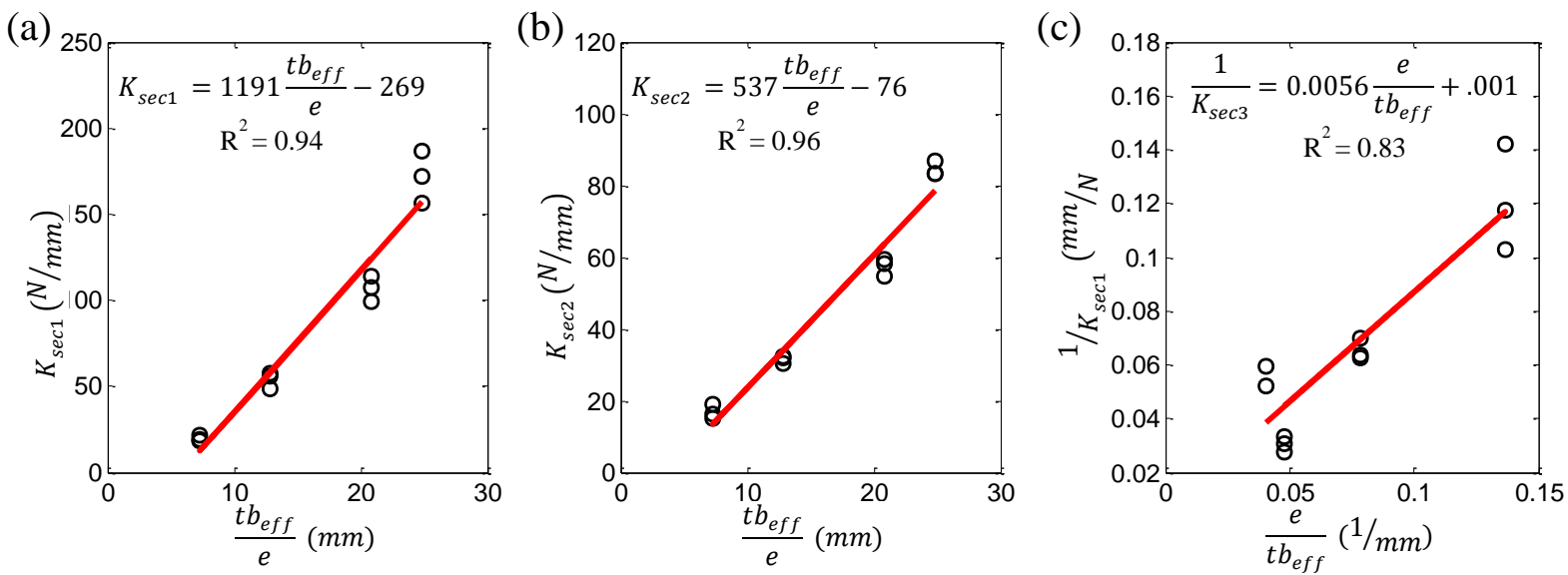

Fig. 16. Fitted lines to secant stiffness values (a) $K_{s e c 1}$, (b) $K_{s e c 2}$, and (c) $K_{s e c 3}$ 
(a)

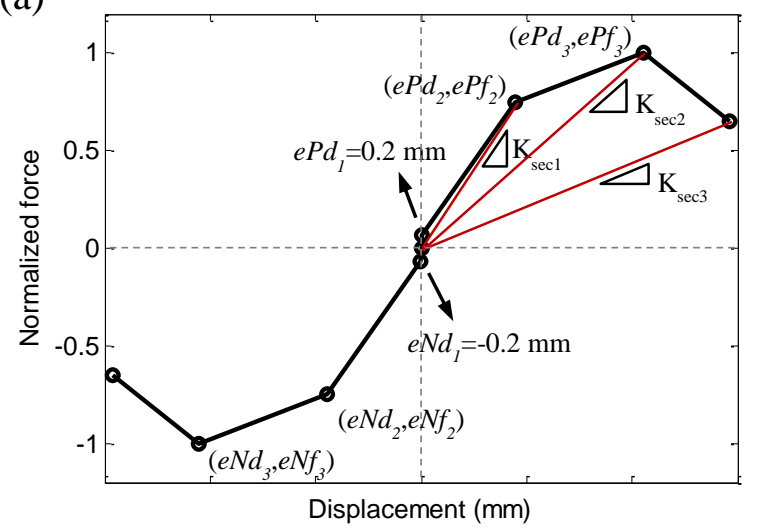

(b)

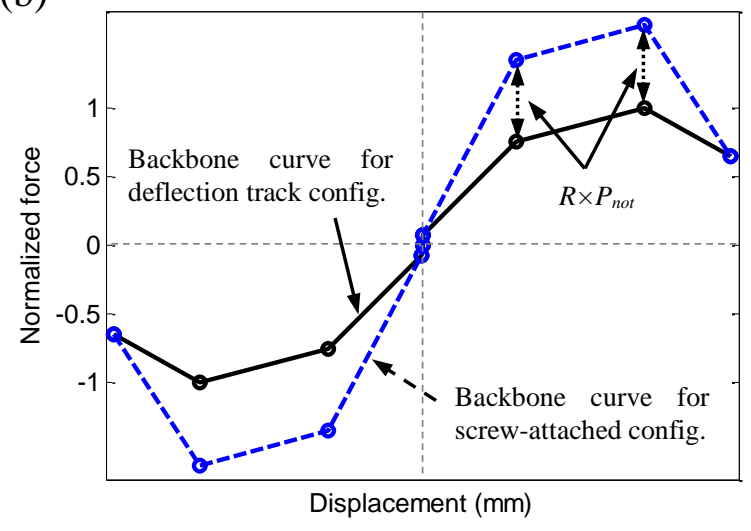

Fig. 17. (a) Normalized generic backbone curve for track-deflection configuration, (b) Schematic modified backbone curve for screw-attached configuration 
Table 1

Test program matrix

\begin{tabular}{lllllll}
\hline Series label & Loading protocol & $\begin{array}{l}\text { Loading rate, } \\
\text { v }(\mathrm{mm} / \mathrm{min} .)\end{array}$ & $\begin{array}{l}\text { Stud/Track } \\
\text { thickness }(\mathrm{mm})\end{array}$ & $\begin{array}{l}\text { Gap } \\
(\mathrm{mm})\end{array}$ & $\begin{array}{l}\text { Connection } \\
\text { configuration }\end{array}$ & $\begin{array}{l}\text { Number of } \\
\text { specimens }\end{array}$ \\
\hline T48G13DT & Monotonic & 0.04 & 0.48 & 13 & Deflection track & 1 \\
& Monotonic & 0.42 & 0.48 & 13 & Deflection track & 1 \\
& Monotonic & 0.21 & 0.48 & 13 & Deflection track & 1 \\
& Cyclic & 0.21 & 0.48 & 13 & Deflection track & 3 \\
T48G03DT & Monotonic & 0.21 & 0.48 & 3 & Deflection track & 1 \\
& Cyclic & 0.21 & 0.48 & 3 & Deflection track & 3 \\
T48G22DT & Monotonic & 0.21 & 0.48 & 22 & Deflection track & 1 \\
& Cyclic & 0.21 & 0.48 & 22 & Deflection track & 3 \\
T48G13SA & Monotonic & 0.21 & 0.48 & 13 & Screw attached & 1 \\
& Cyclic & 0.21 & 0.48 & 13 & Screw attached & 3 \\
T75G13DT & Monotonic & 0.21 & 0.75 & 13 & Deflection track & 1 \\
& Cyclic & 0.21 & 0.75 & 13 & Deflection track & 3 \\
T75G13SA & Monotonic & 0.21 & 0.75 & 13 & Screw attached & 1 \\
& Cyclic & 0.21 & 0.75 & 13 & Screw attached & 3 \\
\hline
\end{tabular}


Table 2

Connection capacity

\begin{tabular}{|c|c|c|c|c|c|c|}
\hline Series label & $\begin{array}{l}\text { Loading } \\
\text { protocol }\end{array}$ & Specimen \# & $\begin{array}{l}\text { Ultimate tested } \\
\text { capacity }\left(\mathrm{P}_{\max }\right), \mathrm{N}\end{array}$ & $\begin{array}{l}\text { Estimated } \\
\text { capacity, } \mathrm{N}\end{array}$ & $\begin{array}{l}\text { Tested/Estimated } \\
\text { capacity ratio }\end{array}$ & $\begin{array}{l}\text { Observed damage } \\
\text { mechanisms }\end{array}$ \\
\hline \multirow[t]{6}{*}{ T48G13DT } & Monotonic & 1 & 671 & 589 & 1.14 & $\mathrm{SD}, \mathrm{ED}, \mathrm{PD}$ \\
\hline & Monotonic & 1 & 689 & 589 & 1.17 & $\mathrm{SD}, \mathrm{ED}, \mathrm{WC}, \mathrm{PD}$ \\
\hline & Monotonic & 1 & 638 & 589 & 1.08 & $\mathrm{SD}, \mathrm{ED}, \mathrm{PD}$ \\
\hline & Cyclic & 1 & 720 & 589 & 1.22 & $\mathrm{SD}, \mathrm{ED}, \mathrm{WC}, \mathrm{PD}$ \\
\hline & Cyclic & 2 & 670 & 589 & 1.14 & $\mathrm{SD}, \mathrm{ED}, \mathrm{PD}$ \\
\hline & Cyclic & 3 & 693 & 589 & 1.18 & $\mathrm{SD}, \mathrm{ED}, \mathrm{PD}$ \\
\hline \multirow[t]{4}{*}{ T48G03DT } & Monotonic & 1 & 1095 & 882 & 1.24 & $\mathrm{SD}, \mathrm{WC}, \mathrm{PD}$ \\
\hline & Cyclic & 1 & 865 & 882 & 0.98 & $\mathrm{SD}, \mathrm{WC}, \mathrm{PD}$ \\
\hline & Cyclic & 2 & 922 & 882 & 1.05 & $\mathrm{SD}, \mathrm{WC}, \mathrm{PD}$ \\
\hline & Cyclic & 3 & 847 & 882 & 0.96 & $\mathrm{SD}, \mathrm{WC}, \mathrm{PD}$ \\
\hline \multirow[t]{4}{*}{ T48G22DT } & Monotonic & 1 & 286 & 337 & 0.85 & $\mathrm{SD}, \mathrm{TD}, \mathrm{PD}$ \\
\hline & Cyclic & 1 & 280 & 337 & 0.83 & $\mathrm{SD}, \mathrm{TD}, \mathrm{PD}$ \\
\hline & Cyclic & 2 & 327 & 337 & 0.97 & $\mathrm{SD}, \mathrm{TD}, \mathrm{PD}$ \\
\hline & Cyclic & 3 & 301 & 337 & 0.89 & $\mathrm{SD}, \mathrm{TD}, \mathrm{PD}$ \\
\hline \multirow[t]{4}{*}{ T48G13SA } & Monotonic & 1 & 1765 & 1571 & 1.12 & $\mathrm{SD}, \mathrm{WC}, \mathrm{SP}, \mathrm{PD}$ \\
\hline & Cyclic & 1 & 1394 & 1571 & 0.89 & SD, WC, SP, PD \\
\hline & Cyclic & 2 & 1559 & 1571 & 0.99 & $\mathrm{SD}, \mathrm{WC}, \mathrm{SP}, \mathrm{PD}$ \\
\hline & Cyclic & 3 & 1667 & 1571 & 1.06 & $\mathrm{SD}, \mathrm{WC}, \mathrm{SP}, \mathrm{PD}$ \\
\hline \multirow[t]{4}{*}{ T75G13DT } & Monotonic & 1 & 983 & 947 & 1.04 & $\mathrm{SD}, \mathrm{TD}, \mathrm{PD}$ \\
\hline & Cyclic & 1 & 1090 & 947 & 1.15 & $\mathrm{SD}, \mathrm{TD}, \mathrm{PD}$ \\
\hline & Cyclic & 2 & 1167 & 947 & 1.23 & $\mathrm{SD}, \mathrm{TD}, \mathrm{PD}$ \\
\hline & Cyclic & 3 & 1144 & 947 & 1.21 & $\mathrm{SD}, \mathrm{TD}, \mathrm{PD}$ \\
\hline \multirow[t]{5}{*}{ T75G13SA } & Monotonic & 1 & 1972 & 1593 & 1.24 & $\mathrm{SD}, \mathrm{WC}, \mathrm{SP}, \mathrm{PD}$ \\
\hline & Cyclic & 1 & 1836 & 1593 & 1.15 & $\mathrm{SD}, \mathrm{WC}, \mathrm{SP}, \mathrm{PD}$ \\
\hline & Cyclic & 2 & 1891 & 1593 & 1.19 & $\mathrm{SD}, \mathrm{WC}, \mathrm{SP}, \mathrm{PD}$ \\
\hline & Cyclic & 3 & 1945 & 1593 & 1.22 & $\mathrm{SD}, \mathrm{WC}, \mathrm{SP}, \mathrm{PD}$ \\
\hline & & & & $\begin{array}{l}\text { Average }= \\
\mathrm{COV}=\end{array}$ & $\begin{array}{l}1.08 \\
0.12\end{array}$ & \\
\hline
\end{tabular}


Table 3

Engineering demand parameters

\begin{tabular}{|c|c|c|c|c|c|}
\hline \multirow[t]{2}{*}{ Series label } & \multirow[t]{2}{*}{$\begin{array}{l}\text { Spec. } \\
\text { No. }\end{array}$} & \multicolumn{3}{|c|}{$\begin{array}{c}\text { Disp., } \\
\text { mm }\end{array}$} & \multirow{2}{*}{$\begin{array}{c}\text { Force, } \\
\mathrm{N} \\
D S 2\end{array}$} \\
\hline & & $D S 1$ & $D S 2$ & $D S 3$ & \\
\hline \multirow[t]{3}{*}{ T48G03DT } & 1 & 1.9 & 11.5 & 47.6 & 865 \\
\hline & 2 & 1.5 & 10.0 & 42.0 & 922 \\
\hline & 3 & 2.3 & 12.0 & 39.6 & 847 \\
\hline \multirow[t]{3}{*}{ T48G13DT } & 1 & 5.8 & 21.7 & 31.5 & 720 \\
\hline & 2 & 5.3 & 21.0 & 28.8 & 669 \\
\hline & 3 & 5.4 & 21.1 & 31.0 & 693 \\
\hline \multirow[t]{3}{*}{ T48G22DT } & 1 & 6.4 & 14.7 & 15.9 & 279 \\
\hline & 2 & 5.1 & 16.9 & 18.2 & 327 \\
\hline & 3 & 6.6 & 15.8 & 21.1 & 301 \\
\hline \multirow[t]{3}{*}{ T48G13SA } & 1 & 4.6 & 28.8 & 39.1 & 1394 \\
\hline & 2 & 5.4 & 26.3 & 44.9 & 1559 \\
\hline & 3 & 6.7 & 26.1 & 39.1 & 1667 \\
\hline \multirow[t]{3}{*}{ T75G13DT } & 1 & 4.0 & 15.2 & 24.6 & 1090 \\
\hline & 2 & 4.4 & 18.3 & 24.6 & 1167 \\
\hline & 3 & 3.1 & 19.0 & 22.8 & 1144 \\
\hline \multirow[t]{3}{*}{ T75G13SA } & 1 & 3.0 & 15.1 & 24.6 & 1836 \\
\hline & 2 & 2.8 & 13.7 & 33.6 & 1891 \\
\hline & 3 & 2.9 & 15.8 & 44.9 & 1945 \\
\hline
\end{tabular}


Table 4

Fragility curve parameters

\begin{tabular}{lllllllll}
\hline \multirow{2}{*}{ Series label } & \multicolumn{1}{l}{$D S 1$} & \multicolumn{3}{c}{$D S 2$} & \multicolumn{3}{c}{$D S 3$} & \multicolumn{3}{c}{$D S 2$} \\
\cline { 2 - 9 } & $\begin{array}{l}\mathrm{x}_{\mathrm{m}}, \\
\mathrm{mm}\end{array}$ & $\beta$ & $\begin{array}{l}\mathrm{x}_{\mathrm{m}}, \\
\mathrm{mm}\end{array}$ & $\beta$ & $\begin{array}{l}\mathrm{x}_{\mathrm{m}}, \\
\mathrm{mm}\end{array}$ & $\beta$ & $\begin{array}{l}\mathrm{x}_{\mathrm{m}}, \\
\mathrm{N}\end{array}$ & $\beta$ \\
\hline T48G03DT & 1.9 & 0.21 & 11.1 & 0.09 & 42.9 & 0.09 & 877 & 0.04 \\
T48G13DT & 5.5 & 0.05 & 21.3 & 0.02 & 30.4 & 0.05 & 694 & 0.04 \\
T48G22DT & 6.0 & 0.14 & 15.8 & 0.07 & 18.3 & 0.14 & 302 & 0.08 \\
T48G13SA & 5.5 & 0.18 & 27.0 & 0.05 & 40.9 & 0.08 & 1536 & 0.09 \\
T75G13DT & 3.8 & 0.18 & 17.4 & 0.12 & 24.0 & 0.04 & 1133 & 0.04 \\
T75G13SA & 2.9 & 0.03 & 14.8 & 0.08 & 33.3 & 0.30 & 1890 & 0.03
\end{tabular}


Table 5

Fixed "Pinching4" parameters

\begin{tabular}{llllllllll}
\hline \multicolumn{10}{c}{ Parameters } \\
\hline rDispP/N & rForceP/N & uForceP/N & $g K$ & gKLimit & $g D$ & $g$ DLimit & $g F$ & $g E$ & dam \\
\hline 0.6 & 0.1 & -0.1 & 0 & 0 & 0 & 0 & 0 & 1 & cycle
\end{tabular}


Table 6

Sample calibrated "Pinching4" backbone parameters

\begin{tabular}{|c|c|c|c|c|c|c|c|c|}
\hline \multirow[t]{2}{*}{ Specimen No. } & \multicolumn{8}{|c|}{$e P f i$ and $e N f i$ in $\mathrm{N}, e P d i$ and $e N d i$ in $\mathrm{mm}$} \\
\hline & $e P f 1$ & $e P f 2$ & $e P f 3$ & $e P f 4$ & eNf1 & eNf2 & eNf3 & eNf4 \\
\hline \multicolumn{9}{|c|}{ Specimen series T48G13AS } \\
\hline \multirow[t]{2}{*}{ Specimen \#1 } & 40 & 934 & 1379 & 489 & -40 & -934 & -1379 & -489 \\
\hline & 0.2 & 7.6 & 21.6 & 35.6 & -0.2 & -7.6 & -21.6 & -35.6 \\
\hline$\ldots$ & $\ldots$ & $\ldots$ & $\ldots$ & $\ldots$ & $\ldots$ & $\ldots$ & $\ldots$ & $\ldots$ \\
\hline \multicolumn{9}{|c|}{ Specimen series T75G13DT } \\
\hline Specimen \#1 & 80 & 934 & 1112 & 734 & -80 & -934 & -1112 & -734 \\
\hline & 0.2 & 9.4 & 20.3 & 24.6 & -0.2 & -9.4 & -20.3 & -24.6 \\
\hline$\cdots$ & $\cdots$ & $\ldots$ & $\ldots$ & $\ldots$ & $\ldots$ & $\ldots$ & $\ldots$ & $\ldots$ \\
\hline
\end{tabular}


Table 7

"Pinching4" backbone parameters for generic models

\begin{tabular}{lllllllll}
\hline \multirow{3}{*}{ Series label } & \multicolumn{7}{c}{$e P f i$ and $e N f i$ in N, $e P d i$ and $e N d i$ in $\mathrm{mm}$} \\
\cline { 2 - 9 } & $e P f 1$ & $e P f 2$ & $e P f 3$ & $e P f 4$ & $e N f 1$ & $e N f 2$ & $e N f 3$ & $e N f 4$ \\
& $e P d 1$ & $e P d 2$ & $e P d 3$ & $e P d 4$ & $e N d 1$ & $e N d 2$ & $e N d 3$ & $e N d 4$ \\
\hline T48G03DT & 40 & 525 & 845 & 513 & -40 & -525 & -845 & -513 \\
& 0.2 & 3.0 & 10.2 & 27.9 & -0.2 & -3.0 & -10.2 & -27.9 \\
T48G13DT & 49 & 498 & 682 & 462 & -49 & -498 & -682 & -462 \\
& 0.2 & 8.9 & 21.1 & 29.2 & -0.2 & -8.9 & -21.1 & -29.2 \\
T48G22DT & 18 & 227 & 274 & 178 & -18 & -227 & -274 & -178 \\
& 0.2 & 11.9 & 17.0 & 18.3 & -0.2 & -11.9 & -17.0 & -18.3 \\
T48G13SA & 40 & 943 & 1496 & 489 & -40 & -943 & -1496 & -489 \\
& 0.2 & 8.1 & 25.4 & 35.6 & -0.2 & -8.1 & -25.4 & -35.6 \\
T75G13DT & 80 & 956 & 1112 & 801 & -80 & -956 & -1112 & -801 \\
& 0.2 & 8.9 & 19.1 & 24.6 & -0.2 & -8.9 & -19.1 & -24.6 \\
T75G13SA & 111 & 1157 & 1715 & 1157 & -111 & -1157 & -1715 & -1157 \\
& 0.2 & 4.1 & 14.7 & 23.4 & -0.2 & -4.1 & -14.7 & -23.4
\end{tabular}


Table 8

"Pinching4" backbone parameters for the generic model for untested connections

\begin{tabular}{llllllll}
\hline \multicolumn{7}{c}{ Normalized $e P f i$ and $e N f i$ (unitless) } \\
\hline$e P f 1$ & $e P f 2$ & $e P f 3$ & $e P f 4$ & $e N f 1$ & $e N f 2$ & $e N f 3$ & $e N f 4$ \\
\hline 0.07 & 0.75 & 1.00 & 0.65 & -0.07 & -0.75 & -1.00 & -0.65
\end{tabular}

